Article

\title{
Profiling Inflammatory Extracellular Vesicles in Plasma and Cerebrospinal Fluid: An Optimized Diagnostic Model for Parkinson's Disease
}

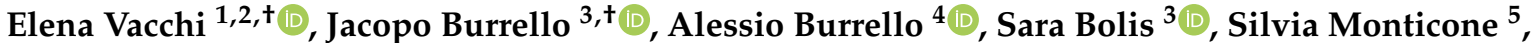 \\ Lucio Barile ${ }^{2,3,6} \mathbb{D}$, Alain Kaelin-Lang ${ }^{1,2,7,8} \mathbb{D}$ and Giorgia Melli 1,2,7,*(D)
}

Citation: Vacchi, E.; Burrello, J.; Burrello, A.; Bolis, S.; Monticone, S.; Barile, L.; Kaelin-Lang, A.; Melli, G. Profiling Inflammatory Extracellular Vesicles in Plasma and Cerebrospinal Fluid: An Optimized Diagnostic Model for Parkinson's Disease. Biomedicines 2021, 9, 230. https:// doi.org/10.3390/biomedicines 9030230

Academic Editors: Andreas Spittler, Wolfgang Holnthoner and Viktoria Weber

Received: 9 February 2021

Accepted: 22 February 2021

Published: 25 February 2021

Publisher's Note: MDPI stays neutral with regard to jurisdictional claims in published maps and institutional affiliations.

Copyright: (c) 2021 by the authors Licensee MDPI, Basel, Switzerland This article is an open access article distributed under the terms and conditions of the Creative Commons Attribution (CC BY) license (https:// creativecommons.org/licenses/by/ $4.0 /)$.
1 Laboratory for Biomedical Neurosciences, Neurocenter of Southern Switzerland, Ente Ospedaliero Cantonale, 6900 Lugano, Switzerland; elena.vacchi@eoc.ch (E.V.); alain.kaelin@eoc.ch (A.K.-L.)

2 Faculty of Biomedical Sciences, Universita della Svizzera Italiana, 6900 Lugano, Switzerland; lucio.barile@cardiocentro.org

3 Laboratory for Cardiovascular Theranostics, Cardiocentro Ticino Institute, Ente Ospedaliero Cantonale, 6900 Lugano, Switzerland; jacopo.burrello@gmail.com (J.B.); sara.bolis@cardiocentro.org (S.B.)

4 Department of Electrical, Electronic and Information Engineering "Guglielmo Marconi", University of Bologna, 40136 Bologna, Italy; alessio.burrello@unibo.it

5 Division of Internal Medicine and Hypertension Unit, Department of Medical Sciences, University of Torino, 10126 Torino, Italy; smv.monticone@gmail.com

6 Institute of Life Sciences, Scuola Superiore Sant'Anna, 56127 Pisa, Italy

7 Department of Neurology, Neurocenter of Southern Switzerland, Ente Ospedaliero Cantonale, 6900 Lugano, Switzerland

8 Department of Neurology, Inselspital, Berne University Hospital, 3010 Berne, Switzerland

* Correspondence: giorgia.melli@eoc.ch; Tel.: +41-(0)918116535; Fax: +41-(0)91-8116915

+ These authors contributed equally to this work.

Abstract: Extracellular vesicles (EVs) play a central role in intercellular communication, which is relevant for inflammatory and immune processes implicated in neurodegenerative disorders, such as Parkinson's Disease (PD). We characterized and compared distinctive cerebrospinal fluid (CSF)derived EVs in PD and atypical parkinsonisms (AP), aiming to integrate a diagnostic model based on immune profiling of plasma-derived EVs via artificial intelligence. Plasma- and CSF-derived EVs were isolated from patients with PD, multiple system atrophy (MSA), AP with tauopathies (AP-Tau), and healthy controls. Expression levels of $37 \mathrm{EV}$ surface markers were measured by a flow cytometric bead-based platform and a diagnostic model based on expression of EV surface markers was built by supervised learning algorithms. The PD group showed higher amount of CSF-derived EVs than other groups. Among the $17 \mathrm{EV}$ surface markers differentially expressed in plasma, eight were expressed also in CSF of a subgroup of PD, 10 in MSA, and 6 in AP-Tau. A two-level random forest model was built using EV markers co-expressed in plasma and CSF. The model discriminated PD from non-PD patients with high sensitivity (96.6\%) and accuracy (92.6\%). EV surface marker characterization bolsters the relevance of inflammation in PD and it underscores the role of EVs as pathways/biomarkers for protein aggregation-related neurodegenerative diseases.

Keywords: plasma; cerebrospinal fluid; extracellular vesicles; machine learning; biomarkers; Parkinson's disease; multiple system atrophy; tauopathies; neuroinflammation

\section{Introduction}

A major current medical need in the field of neurology is the identification of reliable biomarkers in vivo for the diagnosis of neurodegenerative diseases. Parkinson's Disease (PD) is the second most common neurodegenerative disorder of the elderly, characterized by the progressive loss of dopaminergic neurons in the substantia nigra [1]. To date, an effective causal treatment is missing, and the diagnosis still relies exclusively on clinical 
evaluation of motor symptoms that appear late, when approximately $70 \%$ of dopaminergic neurons are already lost [2]. Thus, there is urgent need for accessible and reliable biomarkers that can stratify PD patients in clinical trials for novel therapies. Furthermore, $\mathrm{PD}$ and atypical parkinsonisms (AP) are often misdiagnosed. AP refers to a variety of protein aggregation-related diseases in which patients show signs and symptoms similar to PD but generally do not respond to dopaminergic drug treatment specific for PD and experience a more aggressive course of disease. According to the misfolded protein aggregates present in brain of patients, AP can be distinct in AP with synucleinopathies (AP-Syn), such as multisystem atrophy (MSA) and AP with tauopathies (AP-Tau), such as progressive supranuclear palsy (PSP) and corticobasal degeneration (CBD). The differential diagnosis between PD and AP is challenging [3], preventing an early and appropriate treatment.

Extracellular vesicles (EVs) are emerging as novel and sensitive biomarkers for neurodegenerative diseases $[4,5]$. EVs are a heterogeneous population of membrane particles involved in physiological cell-to-cell communication and transmission of biological signals. EVs are released into body fluids where they are easily accessible. EVs are secreted by many cell types [6-8]: they carry membrane receptors which allow the tracking of the cell of origin [9], while their protein and transcriptomic contents provide information on the cell physio-pathological state $[10,11]$. Central nervous system (CNS) neurons and glia cells release EVs [6] that are able to cross the blood-brain barrier and reach the peripheral blood [11].

In a previous work, we demonstrated that distinctive pools of plasma-derived EV surface markers related to inflammatory and immune cells stratified patients with PD and $\mathrm{AP}$ according to the clinical diagnosis. We characterized distinctive EV subpopulations in plasma by simultaneously immunophenotyping 37 different membrane proteins using a flow cytometry multiplex bead-based platform [12,13] and then a diagnostic model based on the distinctive EV surface proteins profile was generated via supervised machine learning algorithms. The resulting diagnostic model was able to correctly classify $88.9 \%$ of patients, with reliable diagnostic performance after internal and external validations [14]. Indeed, recent studies trying to dissect the cell/tissue origin of circulating EVs, showed that the majority of plasma-derived EVs come from hematopoietic cells, in particular platelets, $\mathrm{B}$ cells and T cells [15]. Thus, in order to increase the sensitivity and specificity of the model for neurodegenerative diseases, we analyzed matched samples of cerebrospinal fluid (CSF) and plasma in a subgroup of patients, assuming that EV surface markers, expressed both in plasma and CSF, may have higher relevance for the respective neurodegenerative disease. In fact, CSF permeates the cerebral cortex, spinal cord, cerebral ventricles, and medullary canal, receiving EVs mainly from the CNS. However, despite its higher specificity for CNS, CSF is less accessible and more difficult to obtain compared with plasma. Moreover, the EV content in CSF is lower and the protocol for extracting EVs is technically more challenging, requiring concentration passages which are not generally required for plasma [16].

Therefore, the aims of the present study were: (1) to characterize CSF-derived EVs in healthy subjects and patients with PD, MSA and AP-Tau; (2) to compare EVs profiling in matched CSF and plasma samples of PD and AP patients; (3) to improve our previous diagnostic model, based on plasma-derived EVs by integrating information provided by CSF-derived EVs immunophenotyping.

\section{Materials and Methods}

\subsection{Subjects}

A total of 16 patients, for which matched plasma and CSF samples were analyzed in the current study, made up the optimization cohort: 4 idiopathic PD, 4 probable MSA and 4 probable AP-Tau, among which 3 had probable progressive supranuclear palsy and 1 had possible corticobasal degeneration. The plasma discovery cohort included 84 subjects (Table S1) [9]. Subjects were recruited from the movement disorder outpatient clinic at Neurocenter of Southern Switzerland in Lugano. Inclusion criteria for MSA, PSP and CBD were based on published diagnostic criteria [17-19]. Each subject underwent blood 
sampling and clinical evaluation, and a subgroup also underwent CSF sampling. Disease gravity was assessed by Hoen and Yahr scale (H\&Y) and Movement Disorder SocietyUnified Parkinson's Disease Rating Scale (MDS-UPDRS); cognitive profile by Mini-Mental State Evaluation (MMSE) and Montreal Cognitive Assessment (MoCA); mood disorder by Beck Depression Inventory II (BDI-II) scale; REM sleep Behavior Disorder (RBD) by RBD screening questionnaire; olfactory function by olfactory test (Burghart Messtechnik $\mathrm{GmbH}$, Wedel, Germany). Levodopa equivalent daily dose (LEDD) was calculated for PD and AP [20]. Patients were excluded from the analysis in case of significant comorbidities: diabetes, renal failure, thyroid pathology, vitamin B12 deficiency, HIV infection, syphilis, coagulopathy, fever, acute or chronic inflammatory diseases, and tumors. Finally, 4 subjects who underwent CSF collection in emergency room for acute headache were included in the study as HC, after CSF analysis showed normal parameters and other pathologies were excluded. The 16 patients for which matched plasma and CSF samples were analyzed in the current study made up the optimization cohort, whereas 84 patients from our previous study [14] made up the plasma discovery cohort and were used to test basic and integrated diagnostic models.

\subsection{Plasma and CSF Preparation}

In total, $10 \mathrm{~mL}$ of blood was collected into anticoagulant-EDTA tubes, after at least $4 \mathrm{~h}$ fasting. The following protocol was performed to obtain EV enriched plasma [21] (Figure 1a): samples were centrifuged for $15 \mathrm{~min}$ at $1600 \times g$ at $10^{\circ} \mathrm{C}$, to eliminate cellular components; then, three consecutive centrifuges were performed to further purify the plasma, eliminating apoptotic bodies and larger EVs $(15 \mathrm{~min}$ at $3000 \times g, 15 \mathrm{~min}$ at $10,000 \times g$ and $30 \mathrm{~min}$ at $20,000 \times \mathrm{g}$ at $4{ }^{\circ} \mathrm{C}$ ). We previously demonstrated no significant change in flowcytometric analysis by MACSPlex assay of plasma samples with and without EV enrichment by ultracentrifugation [14]. Samples were aliquoted and stored at $-80^{\circ} \mathrm{C}$.

Five $\mathrm{mL}$ of CSF was collected into $15 \mathrm{~mL}$ polypropylene tubes, and immediately frozen (Figure 1a). After thawing, $500 \mu \mathrm{L}$ of CSF samples underwent serial centrifuges as plasma samples plus a further ultracentrifugation to maximize EV enrichment $(18 \mathrm{~h}$ at $100,000 \times g$ at $4{ }^{\circ} \mathrm{C}$ ). Pellets were resuspended in $30 \mu \mathrm{L}$ of particle-free PBS. The storage period varied among samples according to the consecutive enrollment of subjects in the study, between 2015-07 and 2020-10.

\subsection{Nanoparticle Tracking Analysis (NTA)}

Nanoparticle concentrations and diameters were measured by NanoSight LM10 (Malvern Panalytical, Malvern, UK) equipped with a $405 \mathrm{~nm}$ laser and Nanoparticle Tracking Analysis NTA 2.3 software (Malvern Panalytical, Malvern, UK). Only for NTA analysis, to rule out a confounding effect of ultracentrifugation on EV amount and diameter, plasma samples $(100 \mu \mathrm{L})$ were centrifuged akin to CSF samples for $18 \mathrm{~h}$ at $100,000 \times g$ at $4{ }^{\circ} \mathrm{C}$. The obtained pellet was resuspended in $100 \mu \mathrm{L}$ of particle-free PBS. A total of $1 \mu \mathrm{L}$ of ultraconcentrated plasma or CSF was diluted in particle-free PBS-1:500 and 1:250, respectively. NTA analyses were performed as previously described [14].

\subsection{MACSPlex Exosome Assay and Flow Cytometry Analysis}

The screening approach (MACSPlex human Exosome Kit, Miltenyi, Bergisch Gladbach, Germany) was previously described $[12,13]$ and is summarized in Figure 1a. The complete list of the $37 \mathrm{EV}$ surface markers that were analyzed is represented in Figure $1 \mathrm{~b}$.

In total, $60 \mu \mathrm{L}$ of plasma and $30 \mu \mathrm{L}$ of ultracentrifuged CSF were added to the MACSPlex Buffer solution (final volume $120 \mu \mathrm{L}$ ) and analyzed with MACSQuant Analyzer-10 flow cytometer (Miltenyi, Bergisch Gladbach, Germany). As a blank control we used MACSPlex Buffer incubated with beads and detection antibodies. Median fluorescence intensity (MFI) for each EV surface marker was normalized by the mean MFI for specific EV markers (CD9, CD63, and CD81). All analyses were based on normalized MFI (nMFI) values. Samples were analyzed blind to the clinical diagnosis. 
Tests for the reliability/specificity of MACSPlex human Exosome Kit for EVs and for the technical consistency and reproducibility of the assay were performed in our previous work [14].

a
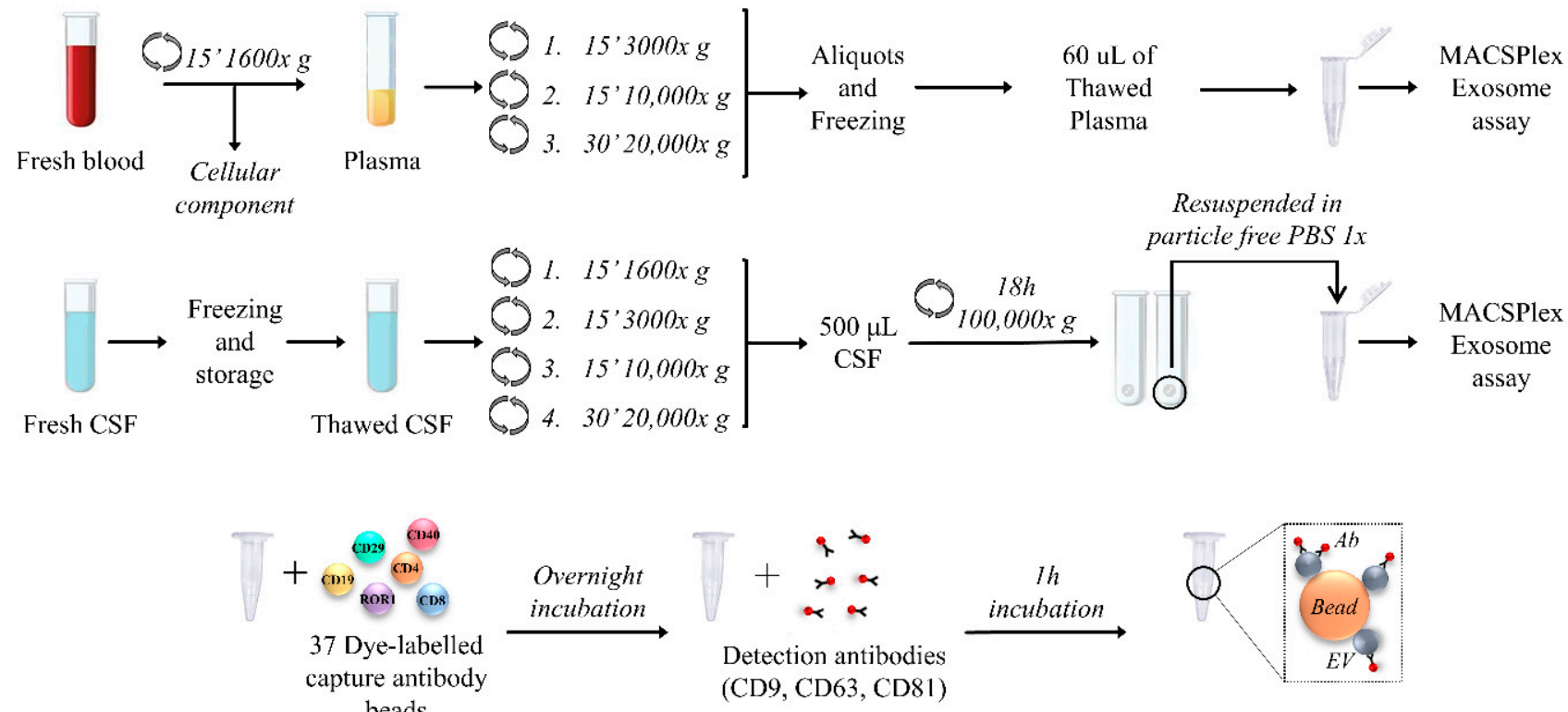

$\mathbf{b}$

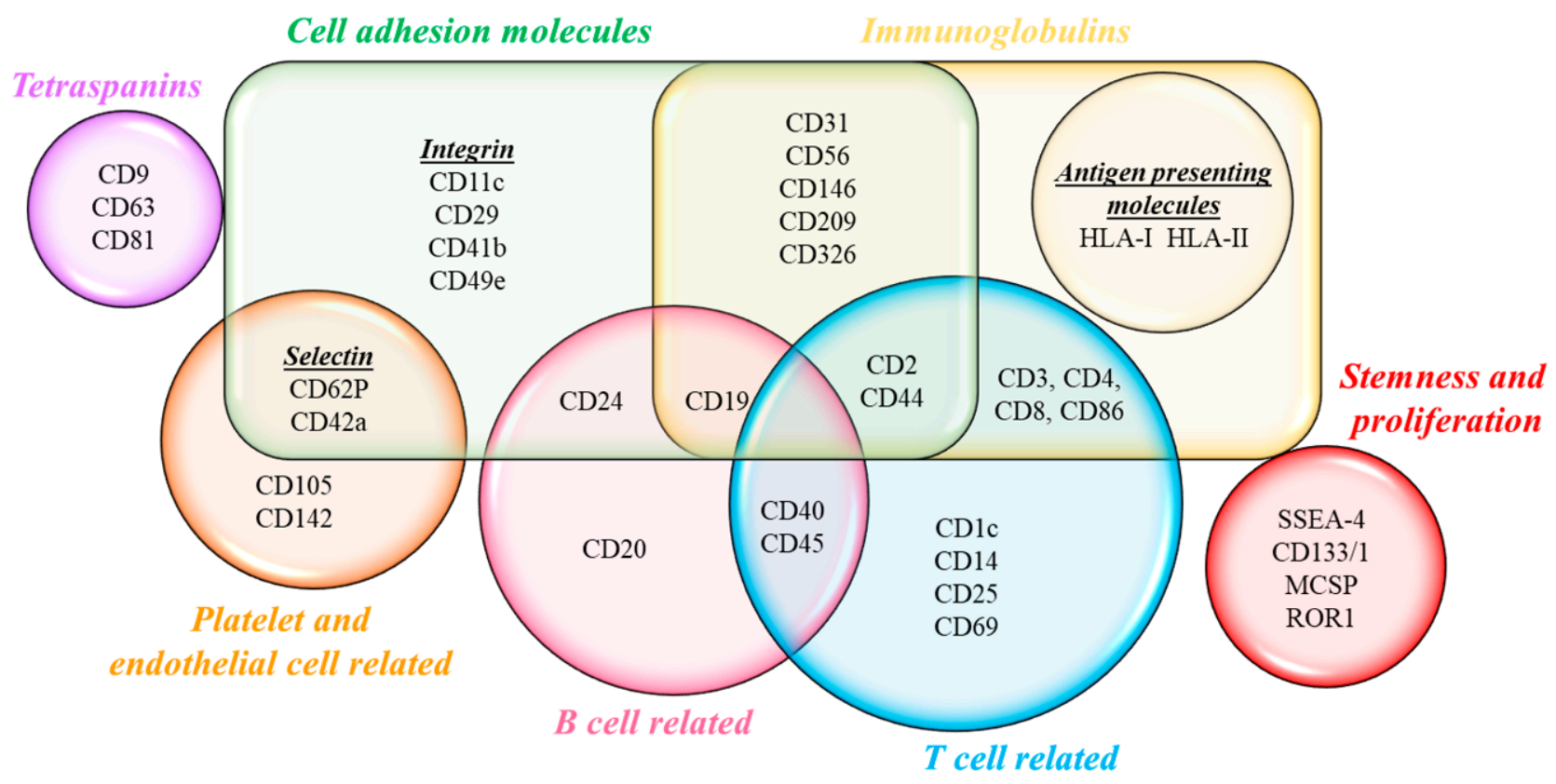

Figure 1. Plasma and CSF preparation for MACSPlex human Exosome assay. (a) Protocol for extracellular vesicle (EV) enrichment and characterization by MACSPlex Exosome Assay. Blood and cerebrospinal fluid (CSF) underwent serial centrifugation to eliminate cellular components and larger EVs. Samples were incubated overnight with phycoerythrin (PE)and fluorescein isothiocyanate (FITC)-labeled capture beads, coated with antibodies against 37 different EV surface markers. APC-conjugated detection antibodies against CD9, CD63, and CD81 were added and incubated for $1 \mathrm{~h}$. After washing steps, samples were analyzed by flow cytometry. (b) Schematic representation of the 37 EV surface markers analyzed by MACSPlex human Exosome assay. 


\subsection{Statistical Analysis}

Statistics was performed using IBM SPSS Statistics 22.0 (IBM SPSS, Armonk, New York, NY, USA), PYTHON 2.7 (Python Software Foundation, DE, USA), and GraphPad PRISM 7.0a (GraphPad Software, San Diego, CA, USA). A Kolmogorov-Smirnov test was applied to evaluated variable distribution. Non-normally distributed variables (disease duration, H\&Y, MDS-UPDRS, BDI-II, MMSE, MoCA, Olfactory test, RBD, LEDD, NTA, MACSPlex analysis) were expressed as medians (interquartile range) and analyzed by Kruskal-Wallis ${ }^{\prime}$ test. Normally distributed variables (age) were expressed as mean \pm standard deviation (SD) and analyzed by 1-way ANOVA test with Bonferroni's correction for multiple comparisons. Categorical variables (sex) were expressed as absolute number and percentage (\%) and analyzed by $\chi^{2}$ (when applicable) or Fisher's tests. Matched measurements (plasma vs. CSF), variables were analyzed by Wilcoxon pairs signed rank test.

\subsection{Diagnostic Modelling}

Supervised learning algorithms were used to combine levels of expression of single EV surface markers in a specific EV surface marker signature and discriminate patients according to clinical diagnosis. Linear discriminant analysis was used as a feature reduction strategy to build the 3D canonical plots (Figure 3d,e); canonical components were calculated from the weighted linear combination of expression levels for the $37 \mathrm{EV}$ markers in order to maximize the separation between the 4 groups (HC, PD, MSA, AP-Tau); each point represents a patient and spheres include patients with a linear combination coefficient that falls within the mean of canonical components 1,2, and $3 \pm$ SD. The diagnostic models were built exploiting a random forest (RF) classification algorithm, as previously reported [14,22,23]. Briefly, each forest is composed of 20 classification trees with a maximum number of 8 splits for each tree; the diagnosis derives from the outcome of each tree of the RF (i.e., if at least 11 of 20 trees of the RF predict PD, the patient will be classified as $\mathrm{PD}$ ).

The basic RF models were built on the expression of $17 \mathrm{EV}$ surface markers (CD29, CD45, CD19, CD42a, HLA-I, CD31, CD1x, CD11c, CD62P, CD40, CD41b, CD209, CD4, CD2, CD146, CD25, MCSP), which were demonstrated to be differentially expressed in plasma samples in HC, PD, MSA, and AP-Tau in our previous study [14]. Internal and external validation of the basic RF models is provided by Vacchi et al. [14].

The integrated version of RF models (see Figure 6) was built selecting only markers expressed in CSF- and plasma-derived EVs from patients with PD (8 EV markers: CD4, CD19, CD2, CD1c, HLA-I, CD41b, CD29, and CD45), MSA (10 EV markers: CD4, CD19, CD2, CD1c, HLA-I, MCSP, CD146, CD41b, CD29, and CD45), or AP-Tau (6 EV markers: CD4, CD2, CD1c, HLA-I, CD41b, and CD45). Integrated models were directly validated on the original discovery cohort. A representative classification tree was shown for each model, and a confusion matrix reported accuracy, sensitivity, specificity, positive and negative predictive values.

\section{Results}

\subsection{Demographic and Clinical Characteristics}

The optimization cohort included 16 patients: 4 PD, 4 MSA, 4 AP-Tau (3 PSP, 1 CBD), and 4 healthy controls (HC). These subjects underwent plasma and CSF collection for paired flow cytometry analysis. Clinical characteristics are summarized in Table 1. No differences were observed between groups ( $p>0.05$ for all comparisons). The plasma discovery cohort included 84 patients from our previous study [14] and was used to test both the basic and integrated diagnostic models (Table S1). In Figure 2, a flowchart of the study is represented. 
Table 1. Clinical characteristics of the cerebrospinal fluid (CSF) optimization cohort.

\begin{tabular}{|c|c|c|c|c|c|}
\hline \multirow[b]{2}{*}{ Variable } & \multirow{2}{*}{$\begin{array}{c}\text { HC } \\
(n=4)\end{array}$} & \multirow{2}{*}{$\begin{array}{c}\text { PD } \\
(n=4)\end{array}$} & \multicolumn{2}{|c|}{ AP } & \multirow[b]{2}{*}{$p$-Value } \\
\hline & & & $\begin{array}{c}\text { MSA } \\
(n=4)\end{array}$ & $\begin{array}{c}\text { AP-Tau } \\
(n=4)\end{array}$ & \\
\hline Age (years) & $59 \pm 20.3$ & $67 \pm 12.9$ & $60 \pm 4.6$ & $66 \pm 12.5$ & 0.794 \\
\hline Sex (ref. male) & $1(25.0)$ & $2(50.0)$ & $1(25.0)$ & $2(50.0)$ & 0.785 \\
\hline Disease duration (years) & - & $\begin{array}{c}4.0 \\
{[0.8-6.5]}\end{array}$ & $\begin{array}{c}3.0 \\
{[1.0-5.8]}\end{array}$ & $\begin{array}{c}3.5 \\
{[3.0-8.5]}\end{array}$ & 0.859 \\
\hline $\mathrm{H} \& \mathrm{Y}$ & - & $\begin{array}{c}1.5 \\
{[1.0-2.8]}\end{array}$ & $\begin{array}{c}3.5 \\
{[2.3-4.8]}\end{array}$ & $\begin{array}{c}4.0 \\
{[2.0-4.0]}\end{array}$ & 0.129 \\
\hline MDS-UPDRS & - & $\begin{array}{c}34.5 \\
{[17.0-51.3]}\end{array}$ & $\begin{array}{c}34.8 \\
{[26.5-34.8]}\end{array}$ & $\begin{array}{c}67.0 \\
{[29.0-67.0]}\end{array}$ & 0.511 \\
\hline Beck Depression & - & $\begin{array}{c}7.0 \\
{[2.3-14.0]}\end{array}$ & $\begin{array}{c}5.5 \\
{[3.5-9.0]}\end{array}$ & $\begin{array}{c}16.0 \\
{[11.0-16.0]}\end{array}$ & 0.094 \\
\hline MMSE & - & $\begin{array}{c}30.0 \\
{[30.0-30.0]}\end{array}$ & $\begin{array}{c}28.0 \\
{[24.5-30.0]}\end{array}$ & $\begin{array}{c}24.0 \\
{[22.0-24.0]}\end{array}$ & 0.051 \\
\hline MOCA & - & $\begin{array}{c}28.0 \\
{[26.3-29.8]}\end{array}$ & $\begin{array}{c}23.0 \\
{[16.3-29.0]}\end{array}$ & $\begin{array}{c}19.0 \\
{[16.0-19.0]}\end{array}$ & 0.114 \\
\hline Olfactory test & - & $\begin{array}{c}6.0 \\
{[3.0-6.0]}\end{array}$ & $\begin{array}{c}9.0 \\
{[4.5-10.5]}\end{array}$ & $\begin{array}{c}7.0 \\
{[5.0-7.0]}\end{array}$ & 0.722 \\
\hline RBD & - & $\begin{array}{c}7.0 \\
{[3.0-8.8]}\end{array}$ & $\begin{array}{c}4.0 \\
{[2.5-4.8]}\end{array}$ & $\begin{array}{c}2.0 \\
{[1.0-2.0]}\end{array}$ & 0.139 \\
\hline LEDD & - & $\begin{array}{c}238 \\
{[100.0-750.0]}\end{array}$ & - & - & - \\
\hline
\end{tabular}

Clinical characteristics of healthy controls (HC) compared to patients diagnosed with Parkinson's disease (PD) multisystem atrophy (MSA), or atypical parkinsonism with tauopathies (AP-Tau). Variables are reported as mean $\pm \mathrm{SD}$, median (interquartile range) and absolute number (percentage), as appropriate. Abbreviations: H\&Y (Hoehn and Yahr scale), MDS-UPDRS (Movement Disorder Society-Unified Parkinson's Disease Rating Scale), BDI-II (Beck Depression Inventory II), MMSE (Mini-Mental State Examination), MoCA (Montreal Cognitive Assessment), RBD (Rem Behavior Disorder scale), and LEDD (LevoDopa equivalent Dose).

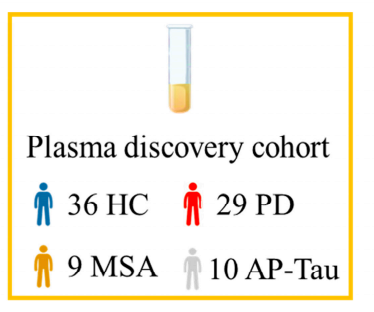

Application on the original
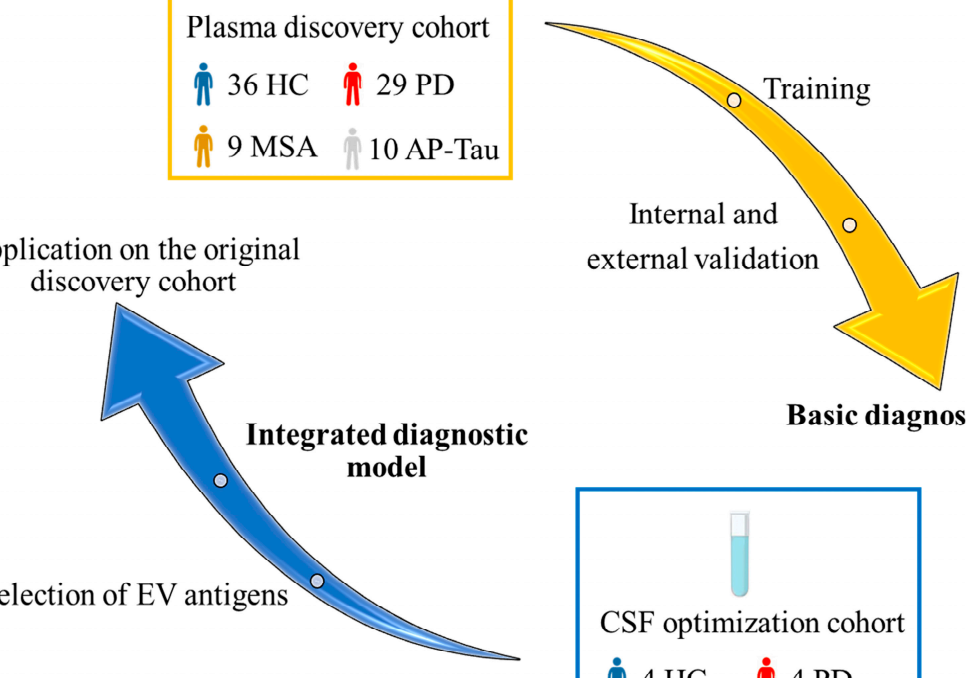

Basic diagnostic model

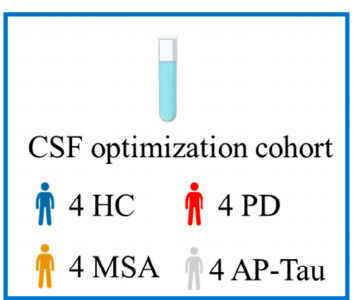

Figure 2. Study flowchart. The plasma discovery cohort was used to train and validate the basic diagnostic model based on plasma-derived extracellular vesicle (EV) profiling. The CSF-derived EV profiling in an optimization cohort was used to generate an integrated model that was subsequently tested in the discovery cohort. 


\subsection{PD Group Shows an Increased Number of CSF-Derived EVS}

Nanoparticle tracking analysis (NTA) revealed higher concentration of CSF-derived nanoparticles $/ \mathrm{mL}$ in PD compared to HC ( $p=0.048)$; this difference was mainly due to larger vesicles (151-500 nm; $p=0.045 ;$ Table S2). A trend towards a higher amount of EVs was observed also in MSA and AP-Tau. Although EV diameter was 1.4/1.5-fold higher in $\mathrm{PD}$ and $\mathrm{AP}$ patients compared to HC, the difference was not significant (Figure $3 \mathrm{~b}$ ).

a

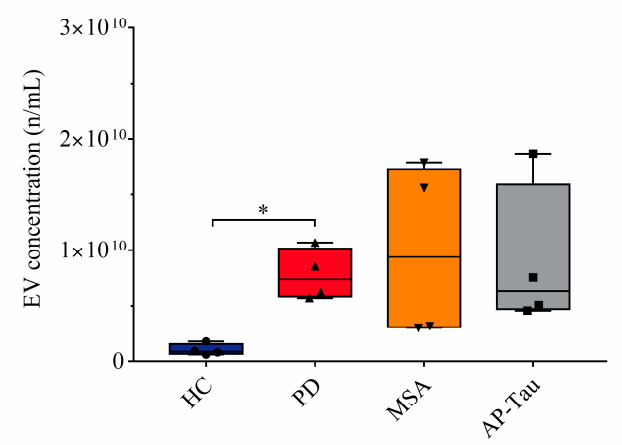

b

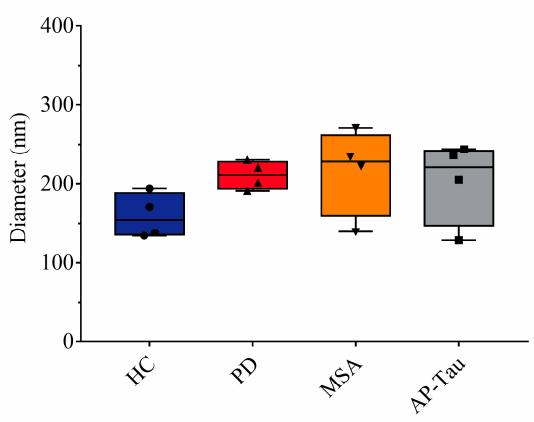

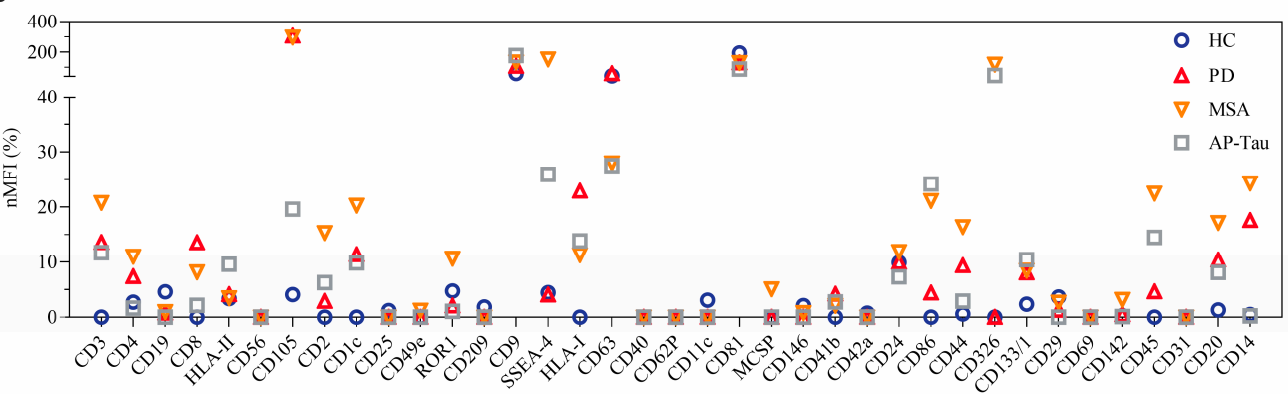

d

e
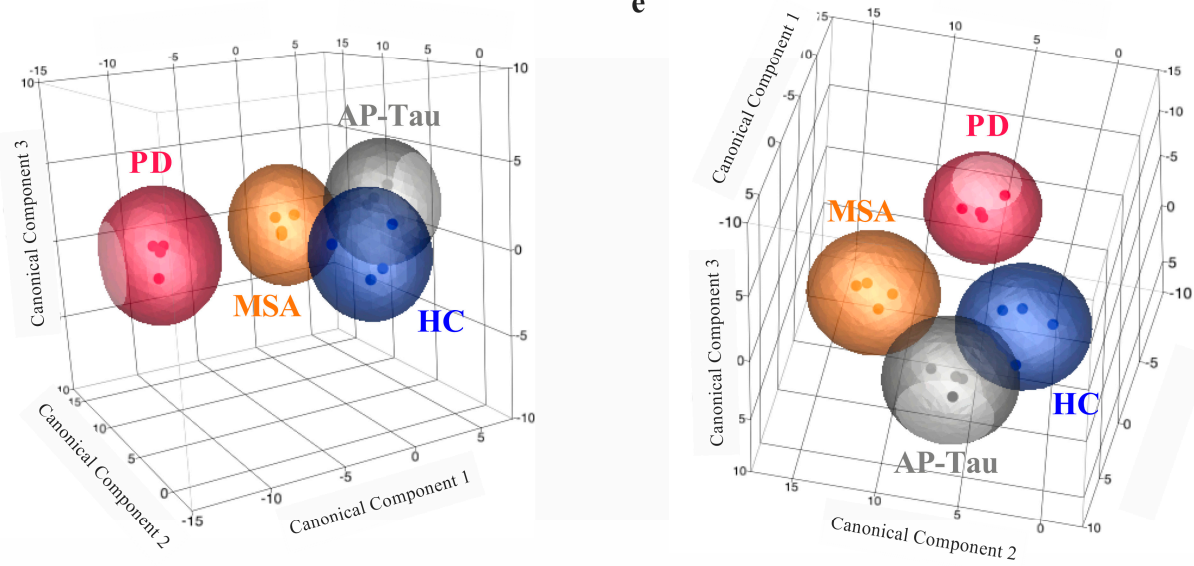

Figure 3. CSF-derived EVs characterization. Characterization of cerebrospinal fluid (CSF)-derived extracellular vesicles (EVs) by nanoparticle tracking analysis (NTA) and MACSPlex human exosome assay flow cytometry. Healthy controls (HC) were compared with patients with Parkinson's disease (PD) multisystem atrophy (MSA), or atypical parkinsonism with tauopathies (AP-Tau). (a) EV concentration (n/mL CSF) at NTA. (b) EV diameter (nm) at NTA. Boxplots show median and interquartile range; bars show minimum and maximum values $\left({ }^{*} p<0.05\right)$. (c) Normalized median fluorescence intensity (nMFI; \%) for $37 \mathrm{EV}$ surface markers. Data and statistics are reported in Table S1. (d,e) Different perspectives of 3D-canonical plot reporting patient discrimination according to EV surface marker expression (each patient is indicated by a point and diagnoses are represented by colors: $\mathrm{HC}$, blue; PD, red; MSA, orange; AP-Tau, grey). Canonical axes of the plot (canonical components 1,2, and 3) are defined by linear discrimination analysis from weighted linear combinations of the $37 \mathrm{EV}$ markers analyzed by flow cytometry. Spheres include patients with linear combination coefficients that fall within the mean $\pm \mathrm{SD}$ (canonicals 1,2 , and $3 \pm \mathrm{SD}$ ). 


\subsection{CSF-Derived EV Immunophenotyping Stratifies Patients According to the Clinical Diagnosis}

The immunophenotyping of 37 surface markers on CSF-derived EVs by MACSPlex assay did not identify statistically significant differences between the four groups when each EV marker was considered individually (Figure $3 \mathrm{c}$ and Table S2). However, several markers, related to T cells (CD2, CD3, CD8, CD14, CD86), B cells (CD20), endothelia cells (CD105) and the major histocompatibility complex class I human leukocyte antigen-ABC (HLA-ABC), displayed higher expressions in PD, MSA, AP-Tau then HC. Nevertheless, the linear weighted combination of all EV markers in a single specific signature by supervised learning (linear discriminant analysis, see methods) allowed the discrimination of patients according to their diagnosis, as shown in the canonical plots (Figure 3d,e).

\subsection{Paired Analysis of CSF vs. Plasma in Patients Identifies Different Amount of EVs and Different Surface Marker Expressions}

We compared plasma- and CSF-derived EVs of the same subjects in pathological groups (PD, MSA, and AP-Tau; $n=12$ ). Plasma samples showed higher numbers of nanoparticles $/ \mathrm{mL}$ compared to CSF, even after stratification for EV diameter $(p<0.001$ for all comparisons; Figure $4 \mathrm{a}$ and Table S3). Moreover, CSF-derived nanoparticles were significantly larger ( $p=0.005$; Figure $4 b$ ). We quantified median fluorescence intensity (MFI) of tetraspanins CD9, CD63 and CD81 (specific markers of EVs) by flow cytometry as an alternative measure of EV concentration. Plasmatic samples had higher level of CD81-MFI and of average MFI for CD9, CD63 and CD81 $(p<0.001)$, compared to CSF (Figure 4c and Table S3). Consistently, nanoparticle concentration by NTA directly correlated to mean MFI for CD9-CD63-CD81 ( $\mathrm{R}=0.552 ; p=0.005)$. Among the $37 \mathrm{EV}$ surface markers analyzed in paired plasma vs. CSF samples, 5 markers were differentially expressed in the PD group, 11 in MSA, and 8 in AP-Tau. All these markers were more highly expressed in plasma than CSF except for CD9 in the MSA group (Figures S1 and S2 and Table S4).

a

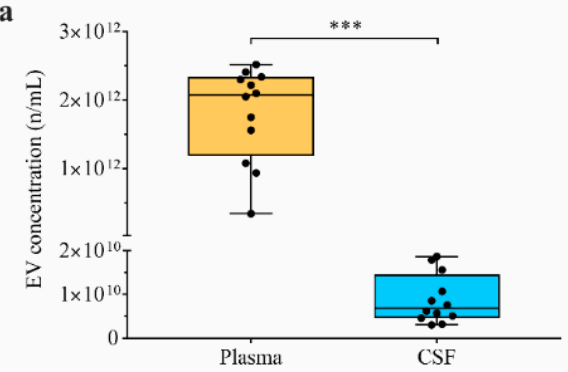

c

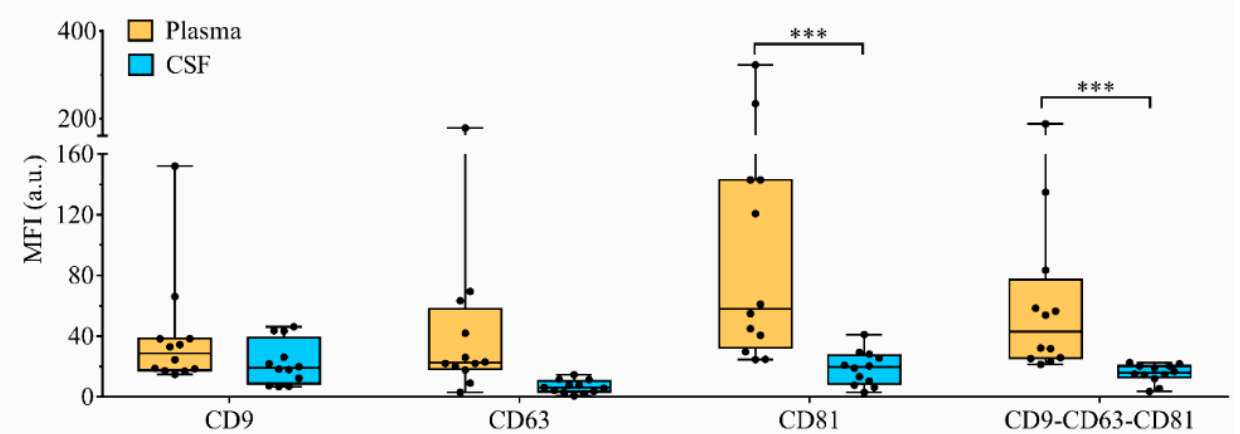

b

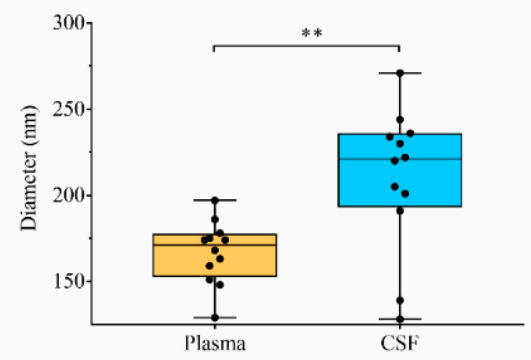

CD9-CD63-CD81

Figure 4. EV quantitative analysis: plasma vs. CSF. Quantitative analysis of extracellular vesicles (EVs) by nanoparticle tracking analysis (NTA) and MACSPlex assay flow cytometry; plasma samples were compared to paired cerebrospinal fluid (CSF) samples in patients with PD (Parkinson's disease; $n=4)$, MSA (multisystem atrophy; $n=4$ ) and AP-Tau (atypical parkinsonism with tauopathies; $n=4)$. (a) EV concentration $(\mathrm{n} / \mathrm{mL}$ plasma or CSF) at NTA. (b) EV diameter (nm) at NTA. (c) MFI (expressed as arbitrary unity; a.u.) for CD9, CD63, CD81 and CD9-CD63-CD81 at flow cytometry. Boxplots show median and interquartile range; bars show minimum and maximum values $\left(^{* *} p<0.05 ;{ }^{* *} p<0.001\right)$. Data and statistics are reported in Table S2. 


\subsection{The Integrated Random Forest Model Demonstrates Higher Diagnostic Accuracy}

In our previous work, 17 plasmatic EV surface markers were differentially expressed in 29 idiopathic PD, 9 probable MSA, 10 probable AP-Tau patients compared to $36 \mathrm{HC}$ (plasma discovery cohort). A random forest (RF) model (we will refer to this model as "basic" throughout the present manuscript) was built combining levels of expression of these EV markers [14]. Among the 17 EV surface markers differentially expressed in the plasma discovery cohort, we selected for each diagnostic group, those which were expressed also in CSF (nMFI different from 0). Eight were expressed also in CSF-derived EVs in patients with PD (CD4, CD19, CD2, CD1c, HLA-I, CD41b, CD29, and CD45), 10 in patients with MSA (CD4, CD19, CD2, CD1c, HLA-I, MCSP, CD146, CD41b, CD29, and CD45), and 6 in patients with AP-Tau (CD4, CD2, CD1c, HLA-I, CD41b, and CD45) (Figure 5). Assuming that EV surface markers expressed both in plasma and CSF may have higher relevance for the respective neurodegenerative disease, we built an "integrated" version of our basic diagnostic model based on plasma EV expression. In particular, we built one model for each type of disease (PD, MSA, AP-Tau). Integrated versions of RF models were subsequently validated in the original plasma discovery cohort (Figure 2).

a

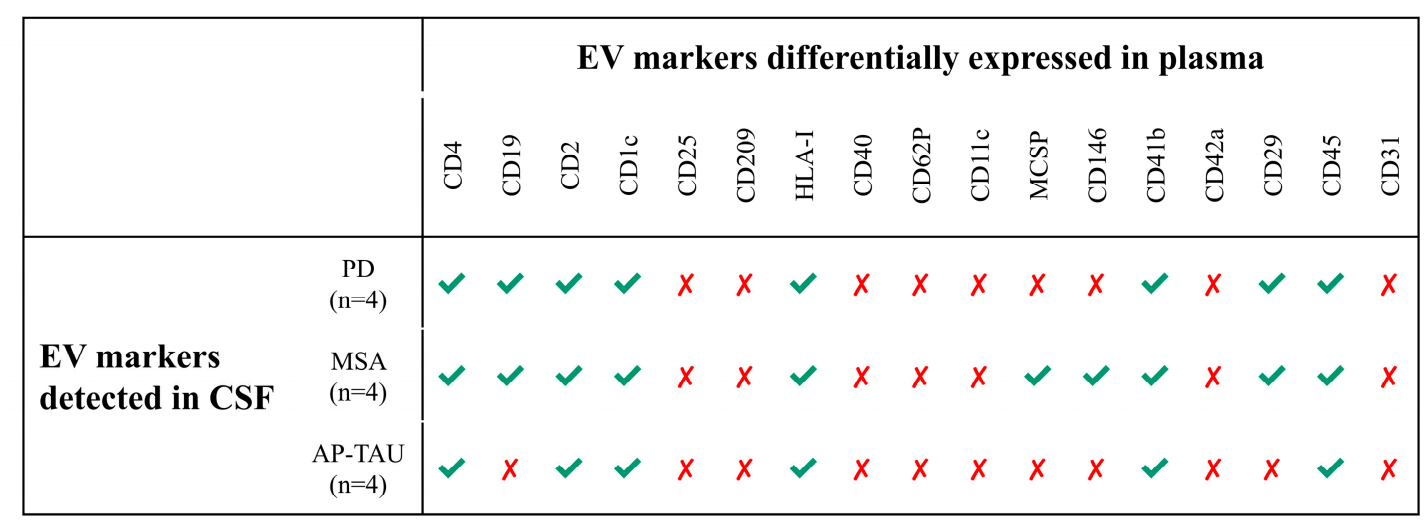

b

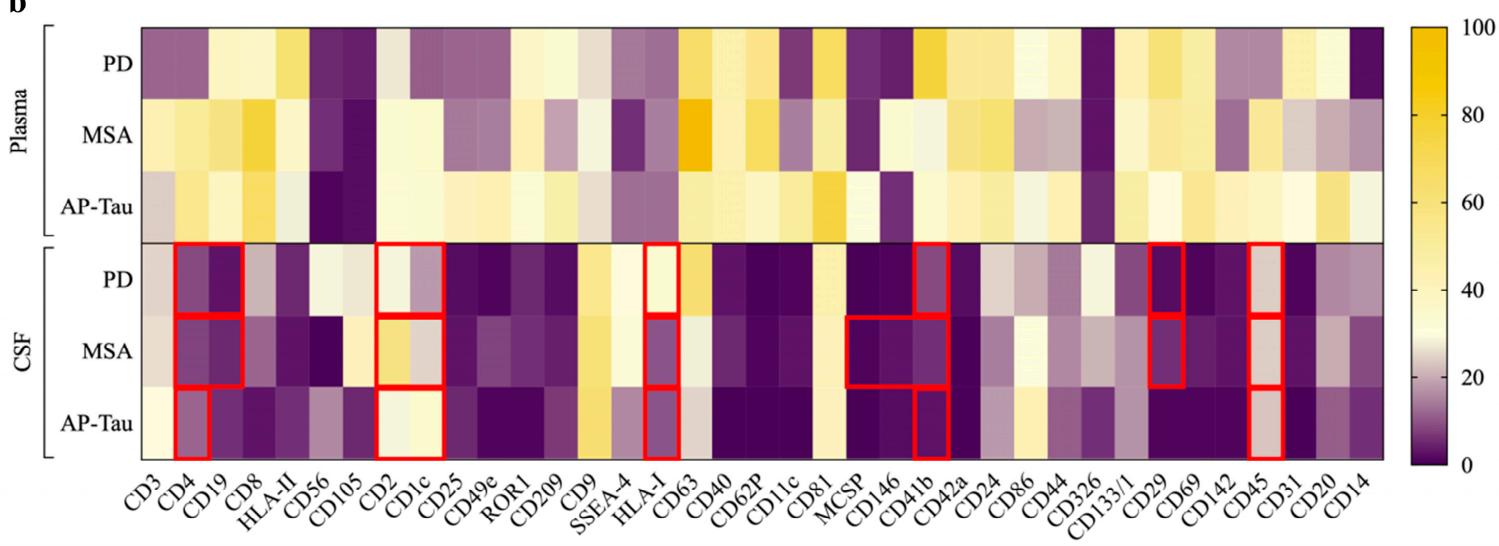

Figure 5. EV surface marker selection. Selection of extracellular vesicle (EV) surface markers for the 2-level random forest diagnostic model: (a) EV surface markers differentially expressed in plasma and detected in cerebrospinal fluid (CSF) of Parkinson's disease (PD), multisystem atrophy (MSA), or atypical parkinsonism with tauopathies (AP-Tau). (b) Heatmap representation of the $37 \mathrm{EV}$ surface markers in plasma and CSF of matched subjects with PD, MSA and AP-Tau. The selected markers in each diagnostic category were highlighted with red boxes.

The level 1 RF basic model [14] (built on the 17 EV surface markers differentially expressed in plasma) was applied to the discovery cohort $(n=84$; Table S1) to differentiate subjects with neurodegenerative diseases from HC (Figure 2). All patients were correctly diagnosed by the model (sensitivity $100.0 \%$ ), whereas 6 of 36 HC were misclassified (specificity $83.3 \%$ ). With an overall accuracy of $92.9 \%$ (Figure 6a), 54 patients (48 patients and 
$6 \mathrm{HC}$ ) were introduced to level 2 analysis, aiming at the selective recognition of PD, MSA, or AP-Tau. The level 2 RF integrated model discriminated PD from non-PD patients (Figure $6 \mathrm{~b}$ ) with higher sensitivity then the RF basic model ( $96.6 \%$ vs. $93.1 \%$, respectively), and increased accuracy $(92.6 \%$ vs. $90.7 \%$, respectively). Similarly, the diagnostic performance was higher in the integrated version for discriminating MSA from non-MSA patients (Figure 6c), with an accuracy of $92.6 \%$ and a sensitivity of $55.6 \%$, while the RF basic model displayed an accuracy and sensitivity of $88.9 \%$ and $33.3 \%$. Finally, the advanced RF model discriminating AP-Tau from non-AP-Tau patients (Figure $6 \mathrm{~d}$ ) correctly predicted 50 of 54 patients $(92.6 \%$ accuracy) with an increased sensitivity $(70.0 \%)$ and a specificity comparable to the basic model ( $97.7 \%)$.
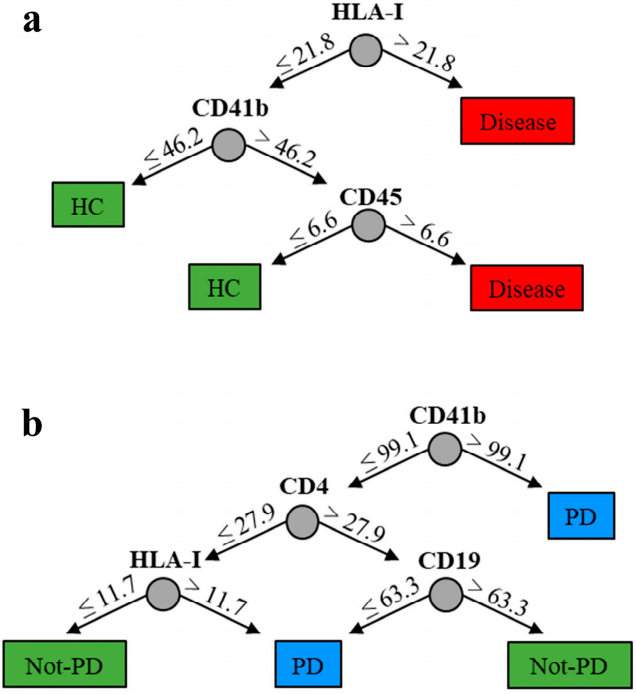

c

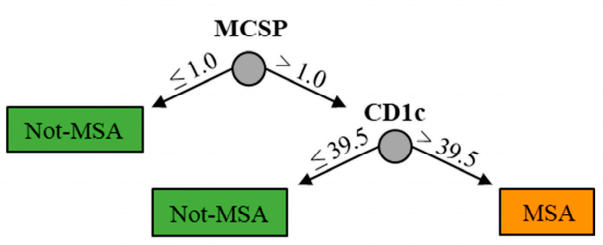

d

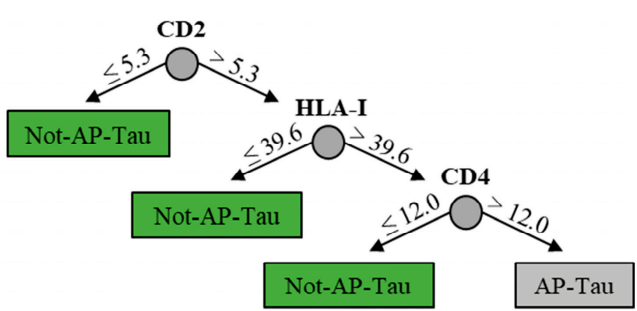

\begin{tabular}{|c|c|c|c|}
\hline \multirow{2}{*}{\multicolumn{2}{|c|}{ Basic }} & \multicolumn{2}{|c|}{ PREDICTED } \\
\hline & & Disease & HC \\
\hline \multirow{2}{*}{ REAL } & Disease & 48 & 0 \\
\hline & $\mathrm{HC}$ & 6 & 30 \\
\hline \multicolumn{4}{|c|}{ Model Accuracy $\mathbf{9 2 . 9 \%}$} \\
\hline \multicolumn{4}{|c|}{ Sensitivity $100.0 \%$} \\
\hline \multicolumn{4}{|c|}{ Specificity $83.3 \%$} \\
\hline \multicolumn{4}{|c|}{ Positive Predictive Value $88.9 \%$} \\
\hline & Negati & ictive Val & $0.0 \%$ \\
\hline
\end{tabular}

Level 1

(Disease $v s . \mathrm{HC}$ )

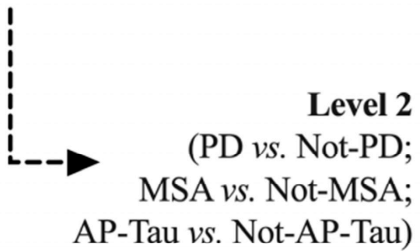

\begin{tabular}{|c|c|cc|}
\hline \multicolumn{2}{|c|}{ Basic } & \multicolumn{2}{c|}{ PREDICTED } \\
\cline { 3 - 4 } REAL & PD & PD & Not-PD \\
\hline & Not-PD & 3 & 2 \\
\hline \multicolumn{3}{|c|}{ Model Accuracy } & $\mathbf{9 0 . 7} \%$ \\
\multicolumn{3}{|c|}{} & Sensitivity $93.1 \%$ \\
\multicolumn{3}{|c|}{ Specificity $88.0 \%$} \\
\multicolumn{2}{|c|}{ Positive Predictive Value $90.0 \%$} \\
\multicolumn{3}{|c|}{ Negative Predictive Value $91.7 \%$} \\
\hline
\end{tabular}

\begin{tabular}{|c|c|c|c|}
\hline \multirow{2}{*}{\multicolumn{2}{|c|}{ Integrated }} & \multicolumn{2}{|c|}{ PREDICTED } \\
\hline & & \multirow{3}{*}{$\begin{array}{c}\text { PD } \\
28 \\
3\end{array}$} & \multirow{2}{*}{$\begin{array}{c}\text { Not-PD } \\
1\end{array}$} \\
\hline PEA & PD & & \\
\hline KEAL & Not-PD & & 22 \\
\hline \multicolumn{4}{|c|}{ Model Accuracy $\mathbf{9 2 . 6 \%}$} \\
\hline \multicolumn{4}{|c|}{ Sensitivity $96.6 \%$} \\
\hline \multicolumn{4}{|c|}{ Specificity $88.0 \%$} \\
\hline \multicolumn{4}{|c|}{ Positive Predictive Value $90.3 \%$} \\
\hline & \multicolumn{3}{|c|}{ Negative Predictive Value $95.7 \%$} \\
\hline
\end{tabular}

\begin{tabular}{|c|c|cc|}
\hline \multicolumn{2}{|c|}{ Basic } & \multicolumn{2}{c|}{ PREDICTED } \\
\cline { 3 - 4 } REAL & MSA & MSA & Not-MSA \\
\hline \multirow{2}{*}{ Not-MSA } & 0 & 6 \\
\hline \multicolumn{3}{|c|}{ Model Accuracy } & $\mathbf{8 8 . 9} \%$ \\
\multicolumn{3}{|c|}{ Sensitivity $33.3 \%$} \\
\multicolumn{3}{|c|}{ Specificity $100.0 \%$} \\
\multicolumn{3}{|c|}{ Positive Predictive Value $100.0 \%$} \\
\multicolumn{3}{|c|}{ Negative Predictive Value $88.2 \%$} \\
\hline
\end{tabular}

\begin{tabular}{|c|c|cc|}
\hline \multirow{2}{*}{ Integrated } & \multicolumn{2}{c|}{ PREDICTED } \\
\cline { 3 - 4 } REAL & MSA & MSA & Not-MSA \\
\hline \multirow{2}{*}{ Not-MSA } & 0 & 4 \\
\hline \multicolumn{3}{|c|}{ Model Accuracy } & $\mathbf{9 2 . 6} \%$ \\
\multicolumn{3}{|r|}{} & Sensitivity $55.6 \%$ \\
& Specificity $100.0 \%$ \\
\multicolumn{3}{|c|}{ Positive Predictive Value $100.0 \%$} \\
\multicolumn{3}{|c|}{ Negative Predictive Value $91.8 \%$} \\
\hline
\end{tabular}

\begin{tabular}{|c|c|c|c|}
\hline \multirow{2}{*}{\multicolumn{2}{|c|}{ Basic }} & \multicolumn{2}{|c|}{ PREDICTED } \\
\hline & & AP-Tau & Not-AP-Tau \\
\hline \multirow{2}{*}{ REAL } & AP-Tau & 5 & 5 \\
\hline & Not-AP-Tau & 0 & 44 \\
\hline \multicolumn{4}{|c|}{ Model Accuracy $\mathbf{9 0 . 7 \%}$} \\
\hline \multicolumn{4}{|c|}{ Sensitivity $50.0 \%$} \\
\hline \multicolumn{4}{|c|}{ Specificity $100.0 \%$} \\
\hline \multicolumn{4}{|c|}{ Positive Predictive Value $100.0 \%$} \\
\hline & Negative $\mathrm{P}$ & dictive Valt & $89.8 \%$ \\
\hline
\end{tabular}

Figure 6. Diagnostic modelling. Development of a 2-level integrated random forest (RF) model to discriminate patients with Parkinson's disease (PD), multisystem atrophy (MSA), or atypical parkinsonism with tauopathies (AP-Tau) in the plasma discovery cohort ( $n=84$; characteristics of patients are reported in Table S4). (a) Level 1 of RF model, discriminating subjects with neurodegenerative diseases from healthy controls (HC). Patients classified as "disease" at level 1 were introduced to level 2. (b) Two-level RF model, discriminating PD from non-PD patients. (c) Two-level RF model, discriminating MSA from non-MSA patients. (d) Two-level RF model, discriminating AP-Tau from non-AP-Tau patients. Confusion matrix (basic vs. integrated version) and a representative classification tree are shown for each RF model. In bold mark are represented the numbers of correctly predicted diagnosis and overall accuracy of the model. 
Overall, the integrated version of the RF model allowed an increased sensitivity in discriminating specific diseases, with higher negative predictive values for all groups of patients.

\section{Discussion}

The main result of this study is the optimization of a diagnostic model for PD, MSA and AP-Tau based on immunophenotyping of plasma-derived EVs by integrating data from CSF-derived EVs via machine learning algorithms. Our previous basic diagnostic model [14], built on $17 \mathrm{EV}$ surface markers differentially expressed in plasma between groups, correctly predicted patient diagnosis in $88.9 \%$ of subjects. The new optimized twolevel RF integrated diagnostic model displayed an overall improved diagnostic accuracy of $92.6 \%$, with an increased sensitivity for all three diagnostic categories with respect to the basic RF model. This result is even more relevant, considering the low numbers of subjects analyzed in this pilot study and opens up the possibility to further improve the accuracy of the model by applying this strategy to larger study groups. Indeed, CSF bears the obvious advantages of being more specific for the CNS environment but at the same time the collecting procedure is more invasive for patients and the protocol to enrich for EVs is more challenging. Thus, our strategy to recalibrate the model on EV surface markers that were expressed both in plasma and CSF in each diagnostic group allowed us to improve the diagnostic system that remains based on EV profiling in plasma. This is a novel approach to blood biomarkers by profiling EV surface markers related to inflammatory and immune cells with potential roles in inflammation related to neurodegeneration, while most of the current studies on EVs as biomarkers are focused especially on evaluating target proteins in neuronal-derived exosomes [24,25]. Further, the multiplexed profiling of inflammation markers allows a more personalized approach, as this biomarker-driven phenotyping might be capable of capturing the clinical heterogeneity of PD and may be used to measure the effect of potential disease-modifying drugs on peripheral inflammatory processes as a proxy for central events. Nevertheless, the direct application of EV immunocapturing and multiarray analysis by flow cytometry to biological fluids (without isolation steps by ultracentrifugation or size exclusion chromatography) has clinical relevance, as it can be achieved in hospital-based diagnostic laboratories. Given its low cost, speed, and simplicity, as well as its high sensitivity and specificity, this approach provides a potential biochemical index to support the clinical assessment of PD and AP. Circulating plasma-derived EVs represent an even more promising tool in characterizing, monitoring, and predicting PD, if their profiling can be achieved avoiding time-consuming protocols and sophisticated instrumentation.

PD patients showed a higher number of EVs in CSF compared to HC, as also demonstrated in plasma [14]. In analogy, MSA and AP-Tau showed a tendency towards higher EV numbers and diameters than HC, but this was not statistically significant, probably due to the low sample size. This result is in line with other observations in patients with Alzheimer's disease where the amount of EVs correlated to myelin damage and neuronal loss [26] and in a mice model of multiple sclerosis in which EVs correlated to brain inflammation, suggesting a pathological role of EVs [27]. Notably, we observed an increase in EVs in neurodegenerative diseases that are typical of elderly populations, while in physiological conditions a decrease in the number of CSF-EVs has been observed by aging [28]. Several human and animal studies have shown that neurodegenerative disorders are characterized by a relevant inflammatory component. PD patients, for example, display neuroinflammatory changes in brain histopathology as well as by elevated immune markers in peripheral blood $[29,30]$, and inflammation has been correlated to the reduction in tyrosine hydroxylase dopaminergic neurons [31] and the expansion of activated microglia in the substantia nigra in animal models of the disease [32]. In an in vitro study, microglial cells activated by proinflammatory stimuli release more microvesicles than exosomes [33]; thus, the higher amount of EVs that we observed in CSF of PD could be related to the inflammatory component. 
Patients with PD, MSA and AP-Tau showed a tendency towards larger EVs in the CSF compared to HC, and moreover, in matched CSF and plasma samples of same patients, CSF-derived EVs showed larger EVs compared to the plasmatic one. Thus, it could be conceivable that in disease groups there is a different pathway of vesicle generation towards production of microvesicles (50-1000 $\mathrm{nm}$ diameters), generated via outward budding of the plasma membrane, rather than exosomes $(40-120 \mathrm{~nm})$ produced in multivesicular bodies via endosomal pathway [34]. Indeed, recent observations suggest that microvesicles may have a role in inflammatory diseases [35] and it has been proposed that they act as a bridge between inflammation and neurodegeneration [36]. An in-depth EV characterization, with different methodologies and with special regard to markers differentially expressed by microvesicles and exosomes, is required to confirm this observation [35].

Regarding the EV immune profiling by MACSPlex human exosome assay applied to CSF-derived EVs, no statistically significant differences were observed in the 37 surface markers between groups. This is certainly due to the main limitation of this explorative study which is the low number of subjects analyzed in each diagnostic category. However, the simultaneous quantification of multiple EV markers increased the power of the assay and displayed different EV profiles of expression in subjects with PD and AP vs. HC, which allowed a good discrimination of patients in accordance with the clinical diagnosis by linear discriminant analysis. Of interest, $\mathrm{PD}$ and $\mathrm{AP}$ patients expressed higher amounts of HLA-I, CD8, CD2, CD3, CD14 and CD20 in CSF, while no or very low detection was observed in $\mathrm{HC}$, suggesting a CNS activation of the immune system, in particular of the $\mathrm{T}$ cell-mediated immunity with particular emphasis on intracellular endogenous synthetized antigens that involves the activation of HLA class I $[37,38]$ and are presented to CD8 T cells [39]. Indeed, a recent work on human substantia nigra demonstrated that CD8 T cell infiltration is an early pathogenic event and parallels the progression of neuronal loss and alpha synuclein aggregation in PD [40]. This is particularly relevant in diseases which are pathologically characterized by intracellular accumulation of misfolded proteins and confirms the crucial role which EVs play in antigen presenting immunity by spreading HLA proteins, possibly increasing the number of dendritic cells or phagocytes presenting them or directly interacting with T cells [41].

CD105 is highly expressed in CSF of PD and MSA. Even if it is used as an endothelial cell marker during angiogenesis [42], it was originally described as a marker for activated macrophages and can identify subtypes of activated microglia [43]. In fact, in the substantia nigra of PD subjects a strong CD105 staining in microglia cells was described in association with degenerating dopaminergic neurons [44]. Activation of microglia is supported also by expression of CD86 in AP-Tau and MSA, whose upregulation had consistently been associated with activated microglia [45], and to a lesser extent in neurons [46] and astrocytes [47], during inflammatory processes.

Finally, levodopa represents the first-line therapy in PD. Several drugs may influence the mechanism of biogenesis and release of EVs [48]; however, to our knowledge, a direct effect of levodopa has never been demonstrated. Indeed, in our previous study, we did not find any significant association when we correlated EV parameters with Levodopa Equivalent Daily Dose (LEDD) in PD and AP subjects [14]. However, larger studies on this topic are certainly recommended.

This is an explorative study and the low number of subjects in the CSF cohort is certainly a limitation; therefore, larger studies and validation in external cohorts of patients are warranted. In addition, it is an antemortem study, lacking the diagnostic confirmation of brain histopathologic analysis. Finally, although it is beyond the primary scope of this paper, a deeper characterization of CSF-derived EVs with different techniques such as transmission electron microscopy (TEM), would be insightful. However, the enrichment of vesicle fraction by immunocapturing displays an inherent limitation for TEM analysis; in fact, a bead-EV complex might impair the ultrastructural morphological characterization of nanosized vesicles. Nevertheless, we can speculate that at least for plasma-derived EVs, 
by avoiding ultracentrifugation, which is known to lead to EV deformation and rapture into smaller particles [49], the morphological structure of immuno-captured EVs is preserved.

In conclusion, a diagnostic model based on plasmatic EVs, built via machine learning, integrating information provided by the simultaneous profiling of CSF and plasma could potentially impact on clinical practice. Furthermore, the EV surface markers characterization bolsters the concept of a relevant involvement of inflammation in PD and it underscores the importance of EVs as pathways/biomarkers for protein aggregationrelated neurodegenerative diseases.

Supplementary Materials: The following are available online at https:/ / www.mdpi.com/2227-9 059/9/3/230/s1, Table S1: Characteristics of patients in plasma discovery cohort; Table S2: NTA and MACSPlex evaluation of CSF-derived EVs; Table S3. EV quantitative analysis: plasma and CSF; Table S4. EV surface antigens in plasma and CSF in pathological groups; Figure S1. EV surface marker expression in plasma and CSF; Figure S2. Evaluation of EV surface antigens in pathological groups.

Author Contributions: Conceptualization: G.M., E.V. and J.B.; methodology: E.V., J.B. and L.B.; software: J.B. and A.B.; validation: J.B. and A.B.; formal analysis: E.V., J.B., A.B. and S.B.; writingoriginal draft preparation: E.V., J.B. and G.M.; writing-review and editing: L.B., S.M. and A.K.-L. All authors have read and agreed to the published version of the manuscript.

Funding: This research was funded by FIDINAM Grant 05.2020 and Swiss Parkinson 08.2020.

Institutional Review Board Statement: The study was conducted according to the guidelines of the Declaration of Helsinki, and approved by the Ticino Cantonal Ethics committee (CE 2895, 19.06.2015).

Informed Consent Statement: Informed consent was obtained from all subjects involved in the study.

Data Availability Statement: Raw data that support the findings of this manuscript are available upon reasonable request to the corresponding author.

Acknowledgments: The authors are grateful to patients and their relatives who participated in this study. They are grateful to Nicole Vago, research nurse, for her valuable work on theclinical database.

Conflicts of Interest: The authors declare no conflict of interest. The funders had no role in the design of the study; in the collection, analyses, or interpretation of data; in the writing of the manuscript, or in the decision to publish the results.

\section{References}

1. Lang, A.E.; Lozano, A.M. Parkinson's disease. Second of two parts. N. Engl. J. Med. 1998, 339, 1130-1143. [CrossRef]

2. Hughes, A.J.; Daniel, S.E.; Kilford, L.; Lees, A.J. Accuracy of clinical diagnosis of idiopathic Parkinson's disease: A clinicopathological study of 100 cases. J. Neurol. Neurosurg. Psychiatry 1992, 55, 181-184. [CrossRef]

3. Tolosa, E.; Wenning, G.; Poewe, W. The diagnosis of Parkinson's disease. Lancet Neurol. 2006, 5, 75-86. [CrossRef]

4. Andjus, P.; Kosanovic, M.; Milicevic, K.; Gautam, M.; Vainio, S.J.; Jagevcic, D.; Kozlova, E.N.; Pivoriunas, A.; Chachques, J.; Sakaj, M.; et al. Extracellular Vesicles as Innovative Tool for Diagnosis, Regeneration and Protection against Neurological Damage. Int. J. Mol. Sci. 2020, 21, 6859. [CrossRef]

5. Pinnell, J.R.; Cui, M.; Tieu, K. Exosomes in Parkinson Disease. J. Neurochem. 2020. [CrossRef]

6. Faure, J.; Lachenal, G.; Court, M.; Hirrlinger, J.; Chatellard-Causse, C.; Blot, B.; Grange, J.; Schoehn, G.; Goldberg, Y.; Boyer, V.; et al. Exosomes are released by cultured cortical neurones. Mol. Cell Neurosci. 2006, 31, 642-648. [CrossRef] [PubMed]

7. Raposo, G.; Stoorvogel, W. Extracellular vesicles: Exosomes, microvesicles, and friends. J. Cell Biol. 2013, 200, 373-383. [CrossRef]

8. Trotta, T.; Porro, C.; Calvello, R.; Panaro, M.A. Biological role of Toll-like receptor-4 in the brain. J. Neuroimmunol. 2014, 268, 1-12. [CrossRef] [PubMed]

9. Thery, C.; Zitvogel, L.; Amigorena, S. Exosomes: Composition, biogenesis and function. Nat. Rev. Immunol. 2002, 2, 569-579. [CrossRef] [PubMed]

10. Fiandaca, M.S.; Kapogiannis, D.; Mapstone, M.; Boxer, A.; Eitan, E.; Schwartz, J.B.; Abner, E.L.; Petersen, R.C.; Federoff, H.J.; Miller, B.L.; et al. Identification of preclinical Alzheimer's disease by a profile of pathogenic proteins in neurally derived blood exosomes: A case-control study. Alzheimers Dement. 2015, 11, 600-607. [CrossRef]

11. Goetzl, E.J.; Boxer, A.; Schwartz, J.B.; Abner, E.L.; Petersen, R.C.; Miller, B.L.; Kapogiannis, D. Altered lysosomal proteins in neural-derived plasma exosomes in preclinical Alzheimer disease. Neurology 2015, 85, 40-47. [CrossRef]

12. Koliha, N.; Heider, U.; Ozimkowski, T.; Wiemann, M.; Bosio, A.; Wild, S. Melanoma Affects the Composition of Blood Cell-Derived Extracellular Vesicles. Front. Immunol. 2016, 7, 282. [CrossRef] 
13. Koliha, N.; Wiencek, Y.; Heider, U.; Jungst, C.; Kladt, N.; Krauthauser, S.; Johnston, I.C.D.; Bosio, A.; Schauss, A.; Wild, S. A novel multiplex bead-based platform highlights the diversity of extracellular vesicles. J. Extracell. Vesicles 2016, 5, 29975. [CrossRef]

14. Vacchi, E.; Burrello, J.; Di Silvestre, D.; Burrello, A.; Bolis, S.; Mauri, P.; Vassalli, G.; Cereda, C.W.; Farina, C.; Barile, L. Immune profiling of plasma-derived extracellular vesicles identifies Parkinson disease. Neurol Neuroimmunol. Neuroinflamm. 2020, 7. [CrossRef]

15. Li, Y.; He, X.; Li, Q.; Lai, H.; Zhang, H.; Hu, Z.; Li, Y.; Huang, S. EV-origin: Enumerating the tissue-cellular origin of circulating extracellular vesicles using exLR profile. Comput. Struct. Biotechnol. J. 2020, 18, 2851-2859. [CrossRef] [PubMed]

16. Street, J.M.; Barran, P.E.; Mackay, C.L.; Weidt, S.; Balmforth, C.; Walsh, T.S.; Chalmers, R.T.A.; Webb, D.J.; Dear, J.W. Identification and proteomic profiling of exosomes in human cerebrospinal fluid. J. Transl. Med. 2012, 10, 1-7. [CrossRef]

17. Armstrong, M.J.; Litvan, I.; Lang, A.E.; Bak, T.H.; Bhatia, K.P.; Borroni, B.; Boxer, A.L.; Dickson, D.W.; Grossman, M.; Hallett, M.; et al. Criteria for the diagnosis of corticobasal degeneration. Neurology 2013, 80, 496-503. [CrossRef]

18. Gilman, S.; Wenning, G.K.; Low, P.A.; Brooks, D.J.; Mathias, C.J.; Trojanowski, J.Q.; Wood, N.W.; Colosimo, C.; Durr, A.; Fowler, C.J. Second consensus statement on the diagnosis of multiple system atrophy. Neurology 2008, 71, 670-676. [CrossRef]

19. Hoglinger, G.U.; Respondek, G.; Stamelou, M.; Kurz, C.; Josephs, K.A.; Lang, A.E.; Mollenhauer, B.; Muller, U.; Nilsson, C.; Whitwell, J.L.; et al. Clinical diagnosis of progressive supranuclear palsy: The movement disorder society criteria. Mov. Disord. 2017, 32, 853-864. [CrossRef] [PubMed]

20. Kipfer, S.; Stephan, M.A.; Schupbach, W.M.; Ballinari, P.; Kaelin-Lang, A. Resting tremor in Parkinson disease: A negative predictor of levodopa-induced dyskinesia. Arch. Neurol. 2011, 68, 1037-1039. [CrossRef]

21. Thery, C.; Amigorena, S.; Raposo, G.; Clayton, A. Isolation and characterization of exosomes from cell culture supernatants and biological fluids. Curr. Protoc. Cell Biol. 2006, 3, 22. [CrossRef]

22. Burrello, J.; Bolis, S.; Balbi, C.; Burrello, A.; Provasi, E.; Caporali, E.; Gauthier, L.G.; Peirone, A.; D'Ascenzo, F.; Monticone, S.; et al. An extracellular vesicle epitope profile is associated with acute myocardial infarction. J. Cell Mol. Med. 2020, 24, 9945-9957. [CrossRef]

23. Castellani, C.; Burrello, J.; Fedrigo, M.; Burrello, A.; Bolis, S.; di Silvestre, D.; Tona, F.; Bottio, T.; Biemmi, V.; Toscano, G.; et al. Circulating extracellular vesicles as non-invasive biomarker of rejection in heart transplant. J. Heart Lung Transplant. 2020, 39, 1136-1148. [CrossRef]

24. Agliardi, C.; Meloni, M.; Guerini, F.R.; Zanzottera, M.; Bolognesi, E.; Baglio, F.; Clerici, M. Oligomeric alpha-Syn and SNARE complex proteins in peripheral extracellular vesicles of neural origin are biomarkers for Parkinson's disease. Neurobiol. Dis. 2021, 148, 105185. [CrossRef]

25. Jiang, C.; Hopfner, F.; Katsikoudi, A.; Hein, R.; Catli, C.; Evetts, S.; Huang, Y.; Wang, H.; Ryder, J.W.; Kuhlenbaeumer, G. Serum neuronal exosomes predict and differentiate Parkinson's disease from atypical parkinsonism. J. Neurol. Neurosurg. Psychiatry 2020, 91, 720-729. [CrossRef] [PubMed]

26. Agosta, F.; Dalla Libera, D.; Spinelli, E.G.; Finardi, A.; Canu, E.; Bergami, A.; Bocchio Chiavetto, L.; Baronio, M.; Comi, G.; Martino, G.; et al. Myeloid microvesicles in cerebrospinal fluid are associated with myelin damage and neuronal loss in mild cognitive impairment and Alzheimer disease. Ann. Neurol. 2014, 76, 813-825. [CrossRef]

27. Verderio, C.; Muzio, L.; Turola, E.; Bergami, A.; Novellino, L.; Ruffini, F.; Riganti, L.; Corradini, I.; Francolini, M.; Garzetti, L.; et al. Myeloid microvesicles are a marker and therapeutic target for neuroinflammation. Ann. Neurol. 2012, 72, 610-624. [CrossRef]

28. Tietje, A.; Maron, K.N.; Wei, Y.; Feliciano, D.M. Cerebrospinal fluid extracellular vesicles undergo age dependent declines and contain known and novel non-coding RNAs. PLoS ONE 2014, 9, e113116. [CrossRef] [PubMed]

29. Sulzer, D.; Alcalay, R.N.; Garretti, F.; Cote, L.; Kanter, E.; Agin-Liebes, J.; Liong, C.; McMurtrey, C.; Hildebrand, W.H.; Mao, X.; et al. T cells from patients with Parkinson's disease recognize alpha-synuclein peptides. Nature 2017, 546, 656-661. [CrossRef]

30. Tansey, M.G.; Romero-Ramos, M. Immune system responses in Parkinson's disease: Early and dynamic. Eur. J. Neurosci. 2019, 49, 364-383. [CrossRef]

31. Reinert, K.R.; Umphlet, C.D.; Quattlebaum, A.; Boger, H.A. Short-term effects of an endotoxin on substantia nigra dopamine neurons. Brain Res. 2014, 1557, 164-170. [CrossRef]

32. Pott Godoy, M.C.; Tarelli, R.; Ferrari, C.C.; Sarchi, M.I.; Pitossi, F.J. Central and systemic IL-1 exacerbates neurodegeneration and motor symptoms in a model of Parkinson's disease. Brain 2008, 131, 1880-1894. [CrossRef] [PubMed]

33. Yang, Y.; Boza-Serrano, A.; Dunning, C.J.R.; Clausen, B.H.; Lambertsen, K.L.; Deierborg, T. Inflammation leads to distinct populations of extracellular vesicles from microglia. J. Neuroinflamm. 2018, 15, 168. [CrossRef]

34. Cocucci, E.; Meldolesi, J. Ectosomes and exosomes: Shedding the confusion between extracellular vesicles. Trends Cell Biol. 2015, 25, 364-372. [CrossRef]

35. Slomka, A.; Urban, S.K.; Lukacs-Kornek, V.; Zekanowska, E.; Kornek, M. Large Extracellular Vesicles: Have We Found the Holy Grail of Inflammation? Front. Immunol. 2018, 9, 2723. [CrossRef]

36. Sankowski, R.; Mader, S.; Valdes-Ferrer, S.I. Systemic inflammation and the brain: Novel roles of genetic, molecular, and environmental cues as drivers of neurodegeneration. Front. Cell Neurosci. 2015, 9, 28. [CrossRef]

37. Guerriero, J.L. Macrophages: Their Untold Story in T Cell Activation and Function. Int. Rev. Cell Mol. Biol. 2019, 342, 73-93. [CrossRef] [PubMed]

38. Stalter, R.D. Chapter 1-MHC Class I. In Measuring Immunity—Basic Biology and Clinical Assessment; Thomson, M., Ed.; Elsevier: New York, NY, USA, 2005; pp. 3-11. 
39. Rock, K.L.; Reits, E.; Neefjes, J. Present Yourself! By MHC Class I and MHC Class II Molecules. Trends Immunol. 2016, 37, 724-737. [CrossRef]

40. Galiano-Landeira, J.; Torra, A.; Vila, M.; Bove, J. CD8 T cell nigral infiltration precedes synucleinopathy in early stages of Parkinson's disease. Brain 2020, 143, 3717-3733. [CrossRef]

41. Bobrie, A.; Colombo, M.; Raposo, G.; Thery, C. Exosome secretion: Molecular mechanisms and roles in immune responses. Traffic 2011, 12, 1659-1668. [CrossRef]

42. Rossi, E.; Bernabeu, C.; Smadja, D.M. Endoglin as an Adhesion Molecule in Mature and Progenitor Endothelial Cells: A Function Beyond TGF-beta. Front. Med. 2019, 6, 10. [CrossRef] [PubMed]

43. Lastres, P.; Bellon, T.; Cabanas, C.; Sanchez-Madrid, F.; Acevedo, A.; Gougos, A.; Letarte, M.; Bernabeu, C. Regulated expression on human macrophages of endoglin, an Arg-Gly-Asp-containing surface antigen. Eur. J. Immunol. 1992, 22, 393-397. [CrossRef] [PubMed]

44. Walker, D.G.; Lue, L.F.; Beach, T.G.; Tooyama, I. Microglial Phenotyping in Neurodegenerative Disease Brains: Identification of Reactive Microglia with an Antibody to Variant of CD105/Endoglin. Cells 2019, 8, 766. [CrossRef]

45. Bechmann, I.; Peter, S.; Beyer, M.; Gimsa, U.; Nitsch, R. Presence of B7-2 (CD86) and lack of B7-1 (CD(80) on myelin phagocytosing MHC-II-positive rat microglia is associated with nondestructive immunity in vivo. FASEB J. 2001, 15, 1086-1088. [CrossRef] [PubMed]

46. Issazadeh, S.; Navikas, V.; Schaub, M.; Sayegh, M.; Khoury, S. Kinetics of expression of costimulatory molecules and their ligands in murine relapsing experimental autoimmune encephalomyelitis in vivo. J. Immunol. 1998, 161, 1104-1112.

47. Zeinstra, E.; Wilczak, N.; De Keyser, J. Reactive astrocytes in chronic active lesions of multiple sclerosis express co-stimulatory molecules B7-1 and B7-2. J. Neuroimmunol. 2003, 135, 166-171. [CrossRef]

48. Cesselli, D.; Parisse, P.; Aleksova, A.; Veneziano, C.; Cervellin, C.; Zanello, A.; Beltrami, A.P. Extracellular Vesicles: How Drug and Pathology Interfere With Their Biogenesis and Function. Front. Physiol. 2018, 9, 1394. [CrossRef]

49. Szatanek, R.; Baran, J.; Siedlar, M.; Baj-Krzyworzeka, M. Isolation of extracellular vesicles: Determining the correct approach (Review). Int. J. Mol. Med. 2015, 36, 11-17. [CrossRef] 